\title{
Observation of self-pulsing in singly resonant optical second-harmonic generation with competing nonlinearities
}

Bache, Morten; Lodahl, Peter; Mamaev, Alexander V.; Marcus, Matt; Saffman, Mark

Published in:

Physical Review A

Link to article, DOI:

10.1103/PhysRevA.65.033811

Publication date:

2002

Document Version

Publisher's PDF, also known as Version of record

Link back to DTU Orbit

Citation (APA):

Bache, M., Lodahl, P., Mamaev, A. V., Marcus, M., \& Saffman, M. (2002). Observation of self-pulsing in singly resonant optical second-harmonic generation with competing nonlinearities. Physical Review A, 65(3), 033811. https://doi.org/10.1103/PhysRevA.65.033811

\section{General rights}

Copyright and moral rights for the publications made accessible in the public portal are retained by the authors and/or other copyright owners and it is a condition of accessing publications that users recognise and abide by the legal requirements associated with these rights.

- Users may download and print one copy of any publication from the public portal for the purpose of private study or research.

- You may not further distribute the material or use it for any profit-making activity or commercial gain

- You may freely distribute the URL identifying the publication in the public portal 


\title{
Observation of self-pulsing in singly resonant optical second-harmonic generation with competing nonlinearities
}

\author{
M. Bache, ${ }^{1,2, *}$ P. Lodahl, ${ }^{1,2, \dagger}$ A. V. Mamaev, ${ }^{1, \$}$ M. Marcus, ${ }^{1}$ and M. Saffman ${ }^{1}$ \\ ${ }^{1}$ Department of Physics, University of Wisconsin, 1150 University Avenue, Madison, Wisconsin 53706 \\ ${ }^{2}$ Optics and Fluid Dynamics Department, Ris $\phi$ National Laboratory, Postbox 49, DK-4000 Roskilde, Denmark
}

(Received 6 November 2000; revised manuscript received 13 August 2001; published 8 February 2002)

\begin{abstract}
We predict and experimentally observe temporal self-pulsing in singly resonant intracavity second-harmonic generation under conditions of simultaneous parametric oscillation. The threshold for self-pulsing as a function of cavity tuning and phase mismatch are found from analysis of a three-component mean-field model. Analytical mean-field calculations of the self-pulsing frequency as well as numerical simulations including the effects of a time-dependent pump pulse agree with the experimentally observed frequencies.
\end{abstract}

DOI: 10.1103/PhysRevA.65.033811

PACS number(s): 42.65.Ky

\section{INTRODUCTION}

In the classic work of McNeil, Drummond, and Walls [1] it was predicted that doubly resonant intracavity secondharmonic generation (SHG) is unstable with respect to highfrequency pulsations of the optical fields. This was one of the first predictions of dynamical instabilities in optical systems [2], and it was subsequently the basis for several theoretical studies with respect to both classical [3] and quantum [4] features of the instability. Despite considerable theoretical interest, there has not, to date, been any experimental observation of this instability. Although, in principle, within reach there are several factors that render observation difficult. One is that the instability has a rather high threshold so that a suitable high power, yet monochromatic, laser source is required. A second challenge is the difficulty of stable operation of the doubly resonant cavity. Self-pulsing was observed in the complementary process of parametric oscillation [5], although it was not possible to make a direct comparison between the measured frequency and theoretical predictions. Furthermore, it was observed recently in an optical parametric oscillator [6] that high pump powers can cause thermal instabilities and pulsations at frequencies orders of magnitude lower than the frequency expected from an intrinsic Hopf bifurcation.

While working towards observing self-pulsing in doubly resonant SHG we discovered self-pulsing in a singly resonant cavity where the fundamental field alone, but not its second harmonic, is resonant. This observation was unexpected since the mean-field equation that describes singly resonant SHG only admits nonoscillatory linear instabilities

\footnotetext{
*Also at IMM, Technical University of Denmark, DK2800 Lyngby, Denmark.

†Present address: Quantum Optics Group, Norman Bridge Laboratory of Physics, California Institute of Technology, Pasadena, CA 91125.

${ }^{\ddagger}$ Permanent address: Institute for Problems in Mechanics, Russian Academy of Sciences, Prospekt Vernadskogo 101, Moscow, 117526 Russia.
}

[7]. We therefore expect that the mechanism leading to the observed self-pulsing is qualitatively different than that found in the doubly resonant cavity.

Indeed, as we describe in this paper, self-pulsing in the singly resonant cavity only occurs due to a competing nonlinear parametric process, where the internally generated second-harmonic field drives a nondegenerate parametric oscillation. This internally pumped optical parametric oscillator (IPOPO) has been the subject of several recent studies [7-10], showing significant modifications to the dynamics of second-harmonic generation, both for classical and quantum effects.

The paper is organized as follows. In Sec. II, we present the analytical model used to explain the observed experimental phenomena, and a linear stability analysis is performed that shows a self-pulsing instability. In Sec. III, the experimental setup is discussed, while Sec. IV is devoted to presenting experimental results and comparing them with results of the linear stability analysis and numerical simulations of the model. The paper is concluded in Sec. V.

\section{THE MODEL}

The derivation of the mean-field model for singly resonant SHG with competing parametric oscillation is described in Ref. [7], and we apply here the same scalings. Since the experimental setup in this paper only concerns temporal instabilities we neglect the effects of diffraction in the crystal, and hence only a single spatial mode is considered. The IPOPO dynamics are then described by the dimensionless set $[7,10]$

$$
\begin{gathered}
\dot{A}_{1}=d_{1} A_{1}+f(\xi)\left|A_{1}\right|^{2} A_{1}+2 h(\xi, \widetilde{\xi}) A_{+} A_{-} A_{1}^{*}+E, \\
\dot{A}_{+}=d_{+} A_{+}+h(\widetilde{\xi}, \xi) A_{1}^{2} A_{-}^{*}+2 f(\widetilde{\xi})\left|A_{-}\right|^{2} A_{+}, \\
\dot{A}_{-}=d_{-} A_{-}+h(\widetilde{\xi}, \xi) A_{1}^{2} A_{+}^{*}+2 f(\widetilde{\xi})\left|A_{+}\right|^{2} A_{-},
\end{gathered}
$$

where the dot denotes differentiation with respect to time. Here $A_{1}$ and $A_{ \pm}$are the amplitudes of the fundamental field (FF) and parametric fields (PFs), respectively, and $E$ is the amplitude of the external pump beam which, without loss of 
generality, is taken to be real. We have defined $d_{j}=-1$ $+i \Delta_{j}, j=1, \pm$, where $\Delta_{1}$ and $\Delta_{ \pm}$are the cavity detunings of the FF and PFs. Due to the symmetry of Eqs. $(1 \mathrm{~b}, \mathrm{c})$ the parametric detunings can be set equal $\Delta_{+}=\Delta_{-}$, and we will in the analysis assume that $\Delta_{1}=\Delta_{+}=\Delta_{-}$that is valid close to degeneracy of the PFs [11]. The second harmonic (SH) at the output of the nonlinear crystal is given by

$$
A_{2}^{\text {out }}=g(\xi) A_{1}^{2}+2 g(\widetilde{\xi}) A_{+} A_{-} .
$$

Finally, the phase-mismatch parameters for the SHG process and the parametric process are $\xi=\left(2 k_{1}-k_{2}\right) L_{c}$ and $\widetilde{\xi}=\left(k_{+}\right.$ $\left.+k_{-}-k_{2}\right) L_{c}$, where $L_{c}$ is the length of the nonlinear medium. In our singly resonant cavity the back mirror induces phase shifts $\theta_{j}, j=1,2, \pm$, on the fields, which enter through the phase-mismatch functions $f, g$, and $h$ given by

$$
\begin{aligned}
& f\left(\xi_{1}\right)=\frac{2 i}{\xi_{1}}+ \frac{e^{-i \xi_{1}}-1}{\xi_{1}^{2}}\left[2+e^{-i 2 \Delta \theta}\left(e^{-i \xi_{1}}-1\right)\right], \\
& g\left(\xi_{1}\right)=e^{i \theta_{2}} \frac{\left(e^{i \xi_{1}}-1\right)\left(e^{i \xi_{1}} e^{i 2 \Delta \theta}+1\right)}{\xi_{1}}, \\
& h\left(\xi_{1}, \xi_{2}\right)= \frac{e^{2 i\left(\xi_{2}-\xi_{1}\right)}-1}{\xi_{2}\left(\xi_{2}-\xi_{1}\right)}+\frac{e^{-i \xi_{1}}-1}{\xi_{1} \xi_{2}} \\
& \times\left[1+e^{i\left(\xi_{2}-\xi_{1}\right)}\left(1-e^{-i 2 \Delta \theta}\right)\right. \\
&\left.+e^{-i \xi_{1}} e^{-i 2 \Delta \theta}\right] .
\end{aligned}
$$

We have here assumed $\theta_{1}=\theta_{+}=\theta_{-}$and introduced $2 \Delta \theta$ $=2 \theta_{1}-\theta_{2}$. For our experimental conditions $\tilde{\xi} \simeq \xi$ as we discuss below, and therefore we use $\xi=\widetilde{\xi}$ in the rest of this paper, unless stated otherwise. In this limit $h(\xi, \xi)=f(\xi)$, which simplifies the theoretical expressions considerably. For low values of the pump $E$ the system is below the parametric threshold $E_{\mathrm{P}}$ and hence the PFs are not excited ( $A_{ \pm}$ $=0)$. In this case the system reduces to a single equation (1a) that, as discussed above, is stable with respect to selfpulsing.

Above the parametric threshold exact analytical solutions of the system can be found as $A_{1}=\bar{A}_{1}, A_{ \pm}=\bar{A}_{ \pm}$. Substituting these into Eqs. (1) it is seen that $\left|\bar{A}_{+}\right|^{2}=\left|\bar{A}_{-}\right|^{2} \equiv I_{0}$. If we now define the fundamental intensity accordingly $I_{1} \equiv\left|\bar{A}_{1}\right|^{2}$, we obtain the set

$$
\begin{gathered}
E^{2}=\zeta_{1}\left(I_{0}\right)+\zeta_{2}\left(I_{1}\right)+\zeta_{3}\left(I_{0}, I_{1}\right), \\
I_{1}^{2}=\frac{4 I_{0}^{2}|f(\xi)|^{2}-4 I_{0}|f(\xi)|\left|d_{1}\right| \cos \left(\phi_{f}-\phi\right)+\left|d_{1}\right|^{2}}{|f(\xi)|^{2}},
\end{gathered}
$$

which can be solved to find $I_{0}$ and $I_{1}$, where $\phi_{f}$ $=\arg [f(\xi)], \phi=-\arctan \left(\Delta_{1}\right)$, and

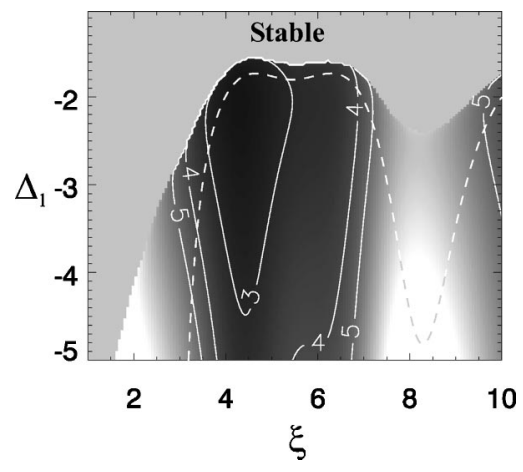

FIG. 1. Contour plot showing the self-pulsing instability for $2 \Delta \theta=-1.5$. The contour lines show different isolevels of the threshold $E_{\mathrm{SP}}$. The dashed line marks the onset of bistability in the homogeneous solutions below the parametric threshold.

$$
\begin{gathered}
\zeta_{1}\left(I_{0}\right)=4 I_{0}\left\{2|f(\xi)| I_{0}\left[\cos \phi_{f}-\Delta_{1} \sin \phi_{f}\right]\right. \\
\left.+\left|d_{1}\right|\left[\Delta_{1} \sin \left(2 \phi_{f}-\phi\right)-\cos \left(2 \phi_{f}-\phi\right)\right]\right\}, \\
\zeta_{2}\left(I_{1}\right)=\left|d_{1}\right|^{2} I_{1}+|f(\xi)|^{2} I_{1}^{3}+2 I_{1}^{2}|f(\xi)|\left(\Delta_{1} \sin \phi_{f}-\cos \phi_{f}\right), \\
\zeta_{3}\left(I_{0}, I_{1}\right)=4|f(\xi)| I_{0} I_{1}\left[\left|d_{1}\right| \cos \left(\phi_{f}-\phi\right)-I_{0}|f(\xi)|\right] .
\end{gathered}
$$

Using the notation $\bar{A}_{1}=\sqrt{I_{1}} e^{i \phi_{A_{1}}, \bar{A}_{ \pm}}=\sqrt{I_{0}} e^{i \phi_{A_{ \pm}}}$, the phases of the exact solutions are found to be

$$
\begin{gathered}
e^{i \phi_{A_{1}}=-}-I_{1}\left[d_{1}^{*}+f^{*}(\xi) I_{1}\right]+2 I_{0} e^{-2 i \phi_{f}}\left[2 f(\xi) I_{0}-\left|d_{1}\right| e^{i \phi}\right], \\
e^{2 i \phi_{A}}=\left[\left|d_{1}\right| e^{i\left(2 \phi_{f}-\phi\right)}-2 I_{0} f(\xi)\right] e^{2 i \phi_{A_{1}}} .
\end{gathered}
$$

Notice that only the sum of the parametric phases can be determined, due to the phase symmetry of Eqs. (1), and therefore in the context of the linear stability analysis the specific choice of the parametric phases remains arbitrary. Here the phases of the PFs are chosen to be identical $\phi_{A}$ $\equiv \phi_{A_{+}}=\phi_{A_{-}}$, and hence the exact parametric solution is given by $\bar{A}_{ \pm}=\sqrt{I_{0}} e^{i \phi_{A}} \equiv \bar{A}$.

The temporal stability of these solutions is tested by perturbing them according to $A_{j}=\bar{A}_{j}+\delta a_{j} e^{\Lambda t}+\delta b_{j} e^{\Lambda * t}, j=1$, \pm . The linearized problem consists of solving a $6 \times 6$ eigenvalue matrix, which is done numerically. Keeping track of the eigenvalue with the largest real part $\Lambda_{\max }$, a self-pulsing Hopf bifurcation occurs when $\operatorname{Re}\left(\Lambda_{\max }\right)=0$ and $\operatorname{Im}\left(\Lambda_{\max }\right)$ $= \pm i \Omega_{c}$. Self-pulsing was found in the region shown in Fig. 1 where $\xi>0$ and $\Delta_{1}<0$. A self-pulsing region also exists for $\xi<0$ and $\Delta_{1}>0$, and will be discussed later. Increasing the pump so $E \gg E_{\mathrm{SP}}$ the system eventually becomes stable again. The parameter area below the dashed line in Fig. 1 is bistable in the homogeneous solutions of the FF when the PFs are not excited $\left(E<E_{\mathrm{P}}, A_{ \pm}=0\right)[7,12]$, which we call the SHG solution. Within this SHG bistable area the upper bistable branch may become self-pulsing unstable when $E$ is increased above $E_{\mathrm{P}}$, and hence hysteresis may occur in the 


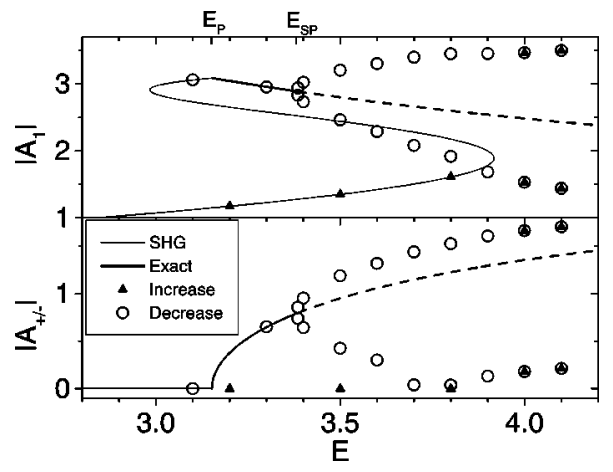

FIG. 2. Homogeneous steady states in the SHG bistable area as calculated in the SHG model without parametric fields (thin) showing bistability and with the exact IPOPO ansatz (thick) setting in at $E=E_{\mathrm{P}}$. Plot calculated for $\Delta_{1}=-3, \xi=6.2$, and $2 \Delta \theta=0$.

self-pulsing behavior. This is shown in Fig. 2 where the thin lines show the SHG bistable solutions. The IPOPO exact solutions (thick lines) becomes valid at $E=E_{\mathrm{P}}$ that is at the upper branch of the SHG bistable solutions, and this branch becomes self-pulsing unstable at $E=E_{\mathrm{SP}}$. A numerical simulation is also shown first increasing $E$ (filled triangles) and then decreasing $E$ (open circles). Increasing $E$ the selfpulsing behavior does not set in at $E_{\mathrm{SP}}$ since the system is still on the lower SHG branch where the parametric fields are not excited. First, when $E$ exceeds the turning point of the SHG bistable branch do the oscillations start (the numerical points now show the minimum and maximum of the oscillations) because here the system jumps to the upper branch, which here is self-pulsing unstable (indicated with dashing). As $E$ is now decreased the upper branch is followed and hence a hysteresis effect in the self-pulsing is observed.

As shown above, the presence of the PFs gives rise to a new instability that is qualitatively different from the SHG instabilities in Ref. [7] since it is a secondary instability above the parametric threshold. New spatiotemporal instabilities due to the PFs were also found in the doubly resonant IPOPO $[11,13]$. On the other hand, as Marte showed in the doubly resonant IPOPO without diffraction [8], the PFs may quench the self-pulsing expected there. Instability quenching is also generic in the doubly resonant IPOPO with diffraction when the PFs are emitted off axis in the case of positive cavity detuning $[11,13]$. Thus, the presence of the PFs can both quench instabilities and give rise to new instabilities.

\section{THE EXPERIMENTAL SETUP}

The self-pulsing instability was observed in the experiment shown in Fig. 3. The nonlinear medium was a $L_{c}$ $=10-\mathrm{mm}$ long by $7 \times 7-\mathrm{mm}$ wide a cut $\mathrm{KNbO}_{3}$ crystal with antireflection coating $(R<0.5 \%)$ at $860 / 430 \mathrm{~nm}$ on both ends. The crystal has index of refraction $n=2.3$ and a nonlinear coefficient measured by single pass $\mathrm{SHG}$ to be $d$ $\simeq 11 \mathrm{pm} / \mathrm{V}$. A linear resonator with planar mirrors was formed between the input coupler $\left(T_{1}=4 \%\right.$ at $860 \mathrm{~nm}, T_{2}$ $=99.8 \%$ at $430 \mathrm{~nm})$ and the back mirror [14] $(R=95 \%$ at $860 / 430 \mathrm{~nm}$ ). The internal round trip losses, not counting the input coupler, were estimated to be $\mathcal{L}=9 \%$ including ab-

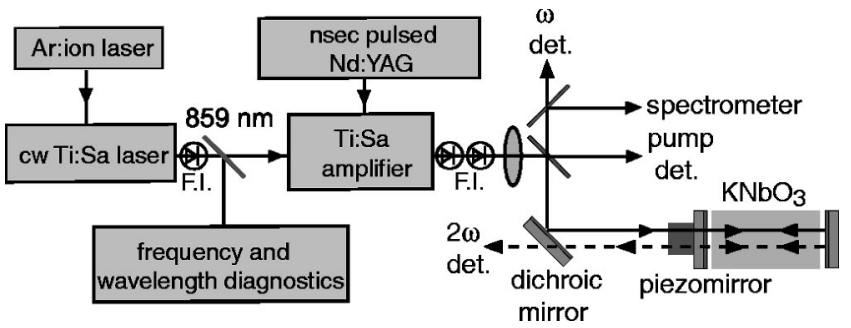

FIG. 3. Experimental geometry.

sorption in the crystal. The total distance $L_{\text {air }}$ between the ends of the crystal and the input coupler and back mirror was $3.0 \mathrm{~mm}$ (1.5 mm at each end). The crystal temperature was adjusted to vary the phase-matching condition and the input coupler was mounted on a piezoelement for fine adjustment of the fundamental cavity tuning $\Delta_{1}$. The cavity was not actively stabilized so the detuning drifted with time.

To generate the pump beam we took the $\lambda_{1}=859 \mathrm{~nm}$ output of a single longitudinal mode ring Ti:Sa laser and amplified it in a multipass geometry by pumping a $2-\mathrm{cm}$ long Ti:Sa rod with 100-mJ pulses from a doubled Nd:Yag laser. The amplifier was operated to give infrared pulses with a peak power of up to about $3 \mathrm{~kW}$ in a roughly $200 \mathrm{~ns}$ [full width at half maximum (FWHM)] long pulse that was focused to a $0.4-\mathrm{mm}$ (FWHM) spot in the nonlinear crystal. Three Faraday isolators, as shown in Fig. 3, were necessary in order to obtain stable operation. The 200-ns pulse corresponds to more than 1000 cavity round trips. The repetition rate of the pulses was $5 \mathrm{~Hz}$, so the average pump power at the cavity was less than $3 \mathrm{~mW}$. Combined with the fact that the pulses are much shorter than the thermal variation time observed in Ref. [6] this suggests that thermal effects in this system are small.

\section{RESULTS}

Here, we will present results obtained from the experimental setup discussed in the preceding section, and use the linear stability analysis from Sec. II and numerical simulations to discuss and confirm these results.

Since we use the IPOPO model to explain the observation of self-pulsing in singly resonant SHG, the presence of the competing parametric beams was verified by dispersing the FF output with a grating (not shown in Fig. 3). From the spectrometer trace shown in Fig. 4 the spectral shift of the parametric fields is $2.6 \mathrm{~nm}$ to each side, which was a typical value. For this spectral shift we estimate from the Sellmeier

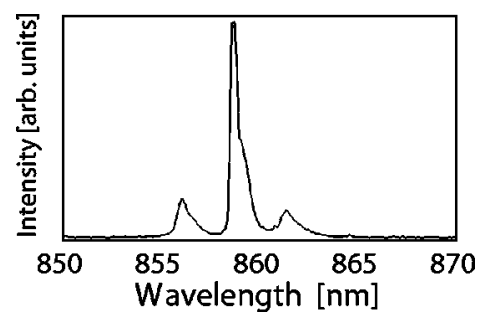

FIG. 4. Spectrometer recording of the parametric fields, located at $856.0 \mathrm{~nm}$ and $861.3 \mathrm{~nm}$, and the fundamental at $858.7 \mathrm{~nm}$. 


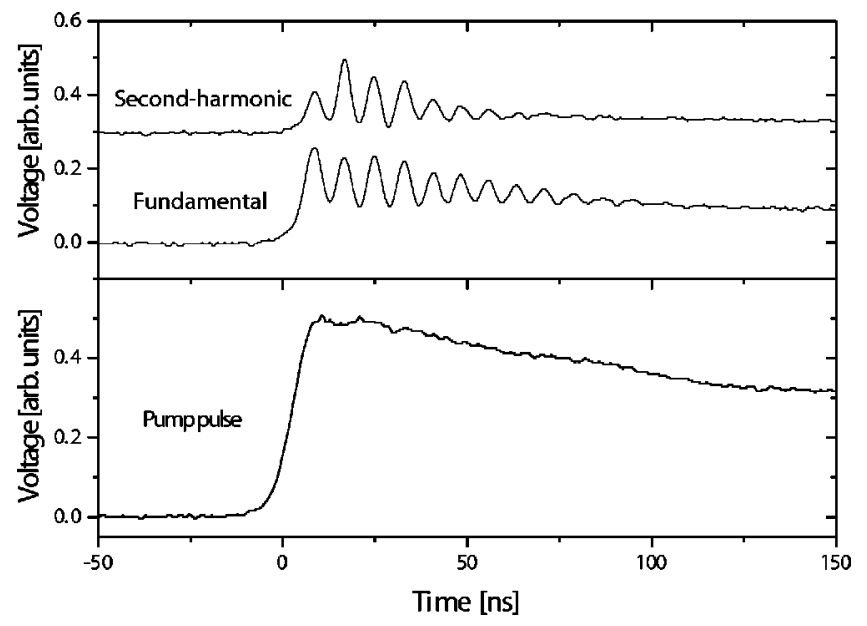

FIG. 5. Self-pulsing observed experimentally in singly resonant SHG for $\xi \simeq 7.3$. Here is shown the intensity of the pump pulse injected into the cavity, and the intensities in the $\mathrm{FF}$ and $\mathrm{SH}$ as measured at the cavity output.

equations that $\widetilde{\xi}-\xi<0.2$, which forms the basis for the approximation $\widetilde{\xi}=\xi$ made in the theory.

Figure 5 presents an experimental observation of selfpulsing for $\xi \simeq 7.3$. After the pump pulse has increased to its maximum value, the $\mathrm{FF}$ and $\mathrm{SH}$ start to oscillate at a regular frequency, and as the level of the pump pulse falls below the self-pulsing threshold again, the oscillations die out $(t$ $\simeq 100 \mathrm{~ns}$ ). A Fourier transform of the experimental data showed a peak around $\nu \simeq 120 \mathrm{MHz}$, while analysis of the individual peaks revealed no direct correlation between the pump level and the oscillation frequency. The intensity threshold in units of $\left[\mathrm{W} / \mathrm{m}^{2}\right]$ is given by

$$
I_{\mathrm{SP}}=E_{\mathrm{SP}}^{2} \frac{\left(T_{1}+\mathcal{L}\right)^{3} \varepsilon_{0} n^{3} c \lambda_{1}^{2}}{64 \pi^{2} T_{1} d^{2} L_{c}^{2}} .
$$

Using the parameters from the experimental setup and the values of $E_{\mathrm{SP}}$ shown in Fig. 1, self-pulsing power thresholds of $P_{\mathrm{SP}}=0.2-8.0 \mathrm{~kW}$ are found $(0.8-3.8 \mathrm{~kW}$ for the experimental values of $\xi$ reported here) which is consistent with the pump power that was available experimentally. Pulse to pulse variations in the pump power that resulted from fluctuations in the laser that pumped the amplifier stage made it difficult to measure the thresholds precisely.

Self-pulsing was observed for a wide range of phasemismatch values, $2<\xi<10$, with frequencies ranging from $80-140 \mathrm{MHz}$, cf., Table I for an overview. In this table, we compare the experimental results with the predictions of the stability analysis based on the following reasonings. The

TABLE I. Frequencies observed experimentally and predicted theoretically for the range of $\Delta_{1}$ shown in Fig. 1.

\begin{tabular}{lccc}
\hline \hline & $\xi=2.1$ & $\xi=7.3$ & $\xi=9.6$ \\
\hline Experiment $\nu(\mathrm{MHz})$ & $105-140$ & $100-135$ & $80-100$ \\
Theory $\nu_{c}(\mathrm{MHz})$ & $165-175$ & $80-110$ & $80-100$ \\
\hline \hline
\end{tabular}
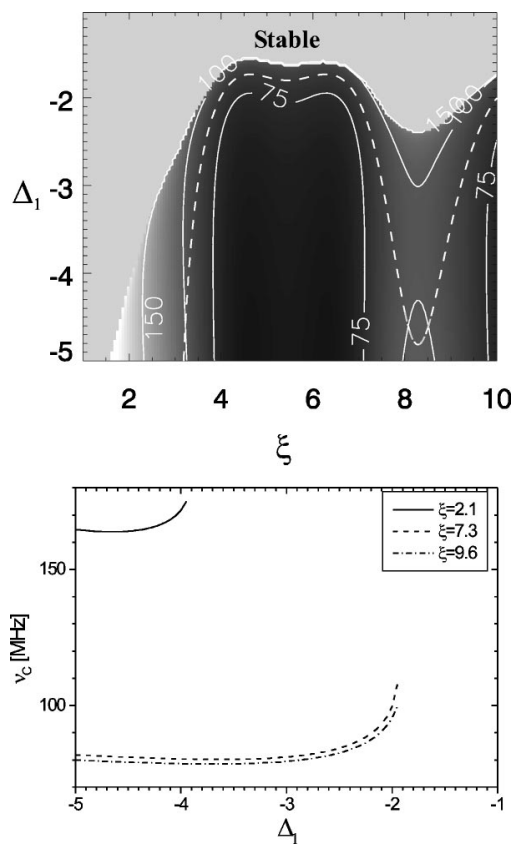

FIG. 6. Contour plot showing the predicted frequency $\nu_{c}$ from the linear stability analysis in $\mathrm{MHz}$ as a function of the detuning $\Delta_{1}$ and the phase mismatch $\xi$. The full lines are isolevels of the frequency while the dashed line again indicates the onset of bistability in the homogeneous solutions below $E_{\mathrm{P}}$. The lower plot shows cuts in the $\xi$ plane for the values of $\xi$ used in the experiment.

time unit of the theoretical model is scaled through the cavity loss rate of the $\mathrm{FF} \gamma_{1}=\left(T_{1}+\mathcal{L}\right) / 2 \tau$, where $\tau=2\left(L_{\text {air }}\right.$ $\left.+n L_{c}\right) / c$ is the cavity round trip propagation time. The predicted oscillation frequency is

$$
\nu=\frac{\gamma_{1}}{2 \pi} \Omega=\frac{c\left(T_{1}+\mathcal{L}\right)}{8 \pi\left(L_{\mathrm{air}}+n L_{c}\right)} \Omega,
$$

where $\Omega$ is the dimensionless Hopf frequency found in the linear stability analysis. Using the above values Eq. (8) gives $\nu \simeq 60 \mathrm{MHz} \times \Omega$. In the experiment the value of $\Delta_{1}$ is unknown and the pump level $E$ varies from shot to shot, while $\xi$ is well known. In Fig. 6 the frequency as calculated from Eq. (8) is shown in a contour plot as a function of $\Delta_{1}$ and $\xi$. Also shown there is the frequency as a function of $\Delta_{1}$ plotted for values of $\xi$ used in the experiment (cuts in the contour plot), and we observe that $\nu$ has only a weak dependence on $\Delta_{1}$ while the dependence on $\xi$ is strong. Moreover, the dependence of $\nu$ on the pump level is also weak, and thus, despite the lack of knowledge of $\Delta_{1}$ or $E$ we may still expect the theory to reasonably predict the observed frequencies. From the figure, we see that the theory predicts values of $\nu_{c}=80-170 \mathrm{MHz}$, and a comparison between theory and experiment can be seen in Table I.

The influence of the pulsed pump was investigated by numerically simulating Eqs. (1) with a temporal pump profile fitted to a typical experimental profile. In Fig. 7, a simulation is shown for $\xi=2.1$ and $\Delta_{1}=-4.0$ and a maximum pump value corresponding to $6 \%$ above threshold, $I_{\mathrm{SP}}$ $=1500 \mathrm{~kW} / \mathrm{cm}^{2}$. In the same figure is shown an experimen- 

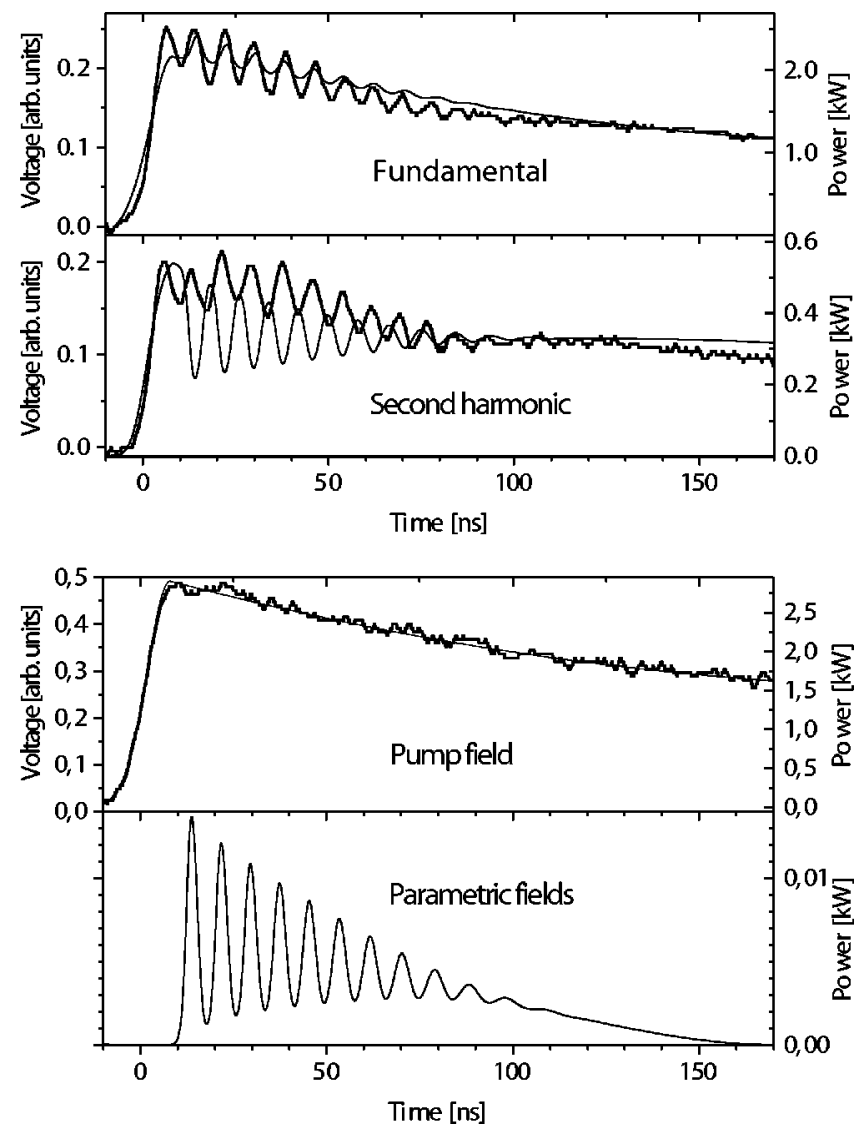

FIG. 7. Time-dependent pump simulations with physical parameters (thin, power axes) along with an experimental trace (thick, voltage axes) showing self-pulsing for $\xi \simeq 2.1$. The simulation is done with $\Delta_{1}=-4.0$ and $I_{\max }=1600 \mathrm{~kW} / \mathrm{cm}^{2} \quad\left(P_{\max }=2.9 \mathrm{~kW}\right)$. The calculated fundamental is the power reflected from the cavity. The lower plot shows the pump profile and the parametric fields observed in the simulation.

tal trace for $\xi \simeq 2.1$, and qualitatively good agreement between numerics and experiment is observed. Fourier transforms of the data gave $\nu \simeq 125 \mathrm{MHz}$ for both experiment and simulation, however, we were not able to find parameters where the amplitudes of the oscillations matched the experiment exactly. In the FF and SH the amplitude in the numerics is too small and too large, respectively, while the stable states reached after $100 \mathrm{~ns}$ match well. The numerical simulations show a phase shift close to $\pi$ between the FF and SH outside the cavity, while only a small phase shift is observed in the experiment. It has been observed in the simulations that the phase difference between the fields depends on various parameters, but we were unable to find parameters of the meanfield model where the phase difference was as seen in the experiment. Additional simulations were performed using $z$-dependent propagation equations instead of the mean-field model (see Ref. [7] for details). These showed similar results as the mean-field model as regards the temporal dynamics and oscillation frequencies. However, the phase difference between the FF and $\mathrm{SH}$ was found to be a sensitive function of cavity detuning, and it was possible to match the experimentally observed phase shift for detunings that were outside the nominal region of validity of the mean-field model. In the

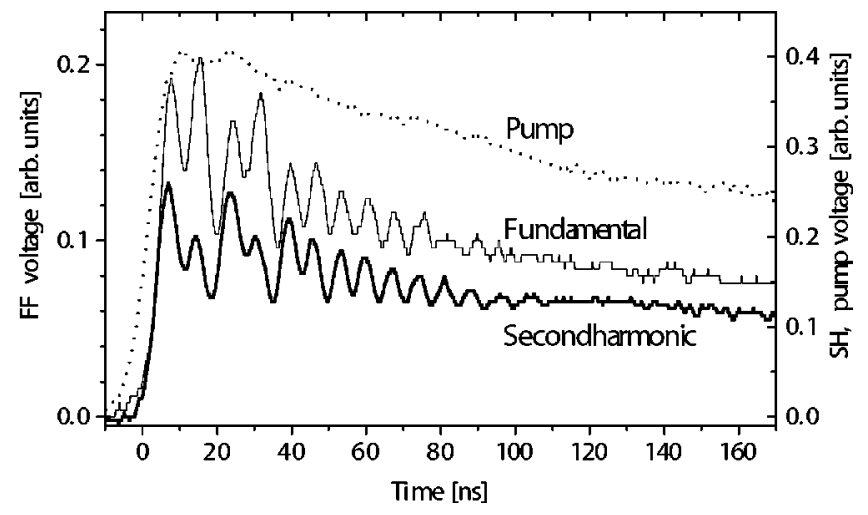

FIG. 8. Self-pulsing with period doubling behavior observed experimentally for $\xi \simeq 2.1$.

simulation $I_{\mathrm{SP}}$ was reached after $40 \mathrm{~ns}$, so the last oscillations are due to relaxation to the stable state. Finally, it should be noted that the levels of the FF and $\mathrm{SH}$ in the experiment cannot be directly compared due to different detector sensitivities. Generally, simulations with the pulsed pump gave up to $30 \%$ lower frequencies than the theory, that is why the frequency observed numerically in Fig. 7 is lower than the theoretical prediction of $\nu_{c}=171 \mathrm{MHz}$. Combining this fact with the values of Table I, for $\xi=7.3$ and $\xi=9.6$ the predicted frequencies are somewhat low, while for $\xi=2.1$ the agreement is good. Further simulations including diffraction and a Gaussian pump profile, as well as cavity propagation instead of a mean-field description, gave similar results.

Even though the model also predicts self-pulsing for negative values of $\xi$, no experimental evidence for this was observed. To understand this recall that the focused beams propagate in a bulk medium so that diffraction is present. The analytical prediction for negative $\xi$ is that the selfpulsing area is located for $\Delta_{1}>0$, and including diffraction in Eqs. (1) we find that the exact solutions for the PFs are here emitted off axis $A_{ \pm}=\bar{A} e^{ \pm i \tilde{\mathbf{k}}_{\perp} \cdot \mathbf{r}}$, where $\left|\widetilde{\mathbf{k}}_{\perp}\right|^{2}=\Delta_{1}$. Performing the analysis allowing for these off-axis solutions showed that self-pulsing does not occur for $\xi<0$, and the same conclusion was reached by performing numerical simulations with physical parameters and a realistic computational window to include diffraction. This strongly indicates that diffraction effectively quenches the instability for $\xi<0$. Note that for $\Delta_{1}<0$ the results of the stability analysis with and without diffraction included are the same, since in both cases the exact solutions are on axis $\left(A_{ \pm}=\bar{A}\right)$. Hence the results presented in Fig. 1 still hold when diffraction is included. Since the system does not show self-pulsing behavior for $\xi<0$ we were here able to observe clamping of the $\mathrm{SH}$, which means that the SH is independent of the pump level. This occurs due to a balance between the competing parametric process and the SHG process, and was first shown experimentally in Ref. [15]. Finally, no self-pulsing was seen when the PFs were not excited in correspondence with the analysis of Ref. [7].

Figure 8 shows an experimental trace for $\xi \simeq 2.1$, where self-pulsing with a frequency of $\nu \simeq 135 \mathrm{MHz}$ is observed. In addition, in the initial part of the pulse where the pump am- 
plitude is highest, we see a more complex behavior with a slow modulation at half the self-pulsing frequency. After approximately $50 \mathrm{~ns}$ only the fast oscillations remain. Such a period doubling, which we also observed in numerical simulations, is possibly an indication of the transition to the kind of multifrequency and chaotic oscillations that have been predicted to occur in doubly resonant SHG at high pump levels [3].

In summary, the analytical results and the numerical simulations back up the results observed experimentally. The discrepancies observed, e.g., the phase difference and the modulation depths, could indicate that the mean-field model is too simple and is, therefore, not well suited to give exact predictions of an experiment this complex.

\section{CONCLUSION}

In conclusion, we have observed self-pulsing in intracavity second-harmonic generation by using a singly resonant setup where only the fundamental was resonated. The instability was explained by the presence of a competing parametric process where the internally generated second-harmonic field drives a nondegenerate parametric oscillation, and experimental evidence of this competing parametric process was presented. The experimentally observed self-pulsing frequency was of order $100 \mathrm{MHz}$ in reasonable agreement with both the analytical and numerical predictions obtained by including the parametric process in a mean-field model. Finally, period doubling behavior in the self-pulsing regime was also observed experimentally.
[1] K.J. McNeil, P.D. Drummond, and D.F. Walls, Opt. Commun. 27, 292 (1978); P.D. Drummond, K.J. McNeil, and D.F. Walls, Opt. Acta 27, 321 (1980).

[2] Self-pulsing in driven optical cavities with different types of nonlinearity is discussed in H. M. Gibbs, Optical Bistability: Controlling Light with Light (Academic Press, Orlando, 1985).

[3] P. Mandel and T. Erneux, Opt. Acta 29, 7 (1982); C.M. Savage and D.F. Walls, ibid. 30, 557 (1983).

[4] P.D. Drummond, K.J. McNeil, and D.F. Walls, Opt. Acta 28, 211 (1981); M.J. Collett and D.F. Walls, Phys. Rev. A 32, 2887 (1985); N.P. Pettiaux, P. Mandel, and C. Fabre, Phys. Rev. Lett. 66, 1838 (1991); O. Veits and M. Fleischhauer, Phys. Rev. A 55, 4516 (1997).

[5] C. Richy et al., J. Opt. Soc. Am. B 12, 456 (1995).

[6] P. Suret et al., Phys. Rev. A 61, 021805 (2000).

[7] P. Lodahl and M. Saffman, Phys. Rev. A 60, 3251 (1999).
[8] M.A.M. Marte, Phys. Rev. A 49, R3166 (1994).

[9] S. Schiller et al., Appl. Phys. Lett. 68, 3374 (1996); A.G. White et al., Phys. Rev. A 55, 4511 (1997); J.L. Sørensen and E.S. Polzik, Appl. Phys. B: Lasers Opt. B66, 711 (1998).

[10] S. Schiller, R. Bruckmeier, and A.G. White, Opt. Commun. 138, 158 (1997).

[11] P. Lodahl, M. Bache, and M. Saffman, Phys. Rev. A 63, 023815 (2001).

[12] A.G. White et al., Europhys. Lett. 35, 425 (1996).

[13] P. Lodahl, M. Bache, and M. Saffman, Opt. Lett. 25, 654 (2000); Phys. Rev. Lett. 85, 4506 (2000).

[14] The internal cavity mirror was dielectric coated silver with a measured relative phase shift of $2 \Delta \theta=-1.5$. Self-pulsing was also observed in a resonator with an optimized back mirror $(2 \Delta \theta \simeq 0)$ coated directly onto the crystal.

[15] K. Schneider and S. Schiller, Opt. Lett. 22, 363 (1997). 